\title{
Diferencias en la variedad de alimentos consumidos por niños preescolares residentes de barrios urbanos de bajos ingresos en ciudades latinoamericanas seleccionadas
}

\author{
Differences in Diversity of Foodstuffs Consumed by \\ Preschool Children Residing in Low-Income Urban \\ Neighborhoods in Selected Latin American Cities
}

\author{
Margarita Fernández A. ${ }^{(1,2)}$, Francisco Lagrutta ${ }^{(3)}$, Marta Cristina Sanabria ${ }^{(4)}$, Rocío Rivera ${ }^{(5)}$, Susana \\ Sánchez Bernal ${ }^{(4)}$, Carlos Castillo-Durán ${ }^{(6)}$
}

\section{RESUMEN}

Introducción: Consumir una escasa variedad de alimentos puede implicar un riesgo de deficiencias o excesos nutritivos en preescolares. Objetivo: Estudiar las diferencias en la variedad de alimentos consumidos por niños preescolares en comunidades de bajos ingresos en ciudades seleccionadas de América Latina. Metodología: Estudio transversal, descriptivo. Las madres completaron una encuesta sobre la variedad de grupos de alimentos consumidos por sus hijos (método de frecuencia de consumo de alimentos) de una lista de los alimentos disponibles en cada mercado local. Participantes: Niños preescolares que asistieron a centros de cuidado médico o jardines escolares $(n=249)$ y residentes en San Salvador (El Salvador), Panamá (Panamá), Asunción (Paraguay) y Santiago (Chile). Resultados: El número promedio de alimentos disponibles en el mercado local estuvo en un rango de 111 a 177; sin embargo el número de alimentos consumidos por los pre escolares, de acuerdo a la estación o en ocasiones, tuvo un rango de 65 a 86, siendo mayor la variedad de alimentos consumidos en San Salvador, más que en las demás ciudades (ANOVA, $\mathrm{p}<0.0001)$. En relación a la proporción de alimentos disponibles para cada grupo de alimentos, las frutas fueron consumidas entre el 44-65\%, el grupo de hortalizas entre 34$56 \%$, y el grupo de pescados-mariscos entre $8-46 \%$. No hubo diferencias en el número promedio de alimentos

\begin{abstract}
Introduction: Consumption of a limited variety of foodstuffs can entail a risk of nutritional deficiencies or excesses in preschool children. Objective: To study differences in the diversity of foodstuffs consumed by preschool children in low-income communities in selected cities in Latin America. Methodology: We conducted a descriptive cross-sectional study.Mothers completed a survey about the variety of foods consumed by their children (foodstuff-consumption frequency method) from a list of foodstuffs available in their respective local markets. Participants: Preschool children who presented at medical treatment centers or attended kindergartens in, and were residents of, San Salvador (El Salvador); Panama City (Panama); Asuncion (Paraguay); and Santiago (Chile) were included. Results: The number of single food items available per city ranged between 111 and 177; those consumed by children either usually, seasonally or occasionally, ranged between 65 and 86, higher in San Salvador than in the other cities (ANOVA, $\mathrm{p}<0.0001$ ). As a proportion of foods available for every group of foods, fruits consumed ranged between $44-65 \%$, vegetables between $34-56 \%$ and fish-sea foods between $8-46 \%$. Additionally, no significant differences in the amount of foods consumed was found between healthy, overweight or undernourished children. Conclusions:The preschool
\end{abstract}

1. Instituto de Nutrición y Tecnología de los Alimentos (INTA), Universidad de Chile. Chile.

2. Universidad del Atlántico. Barranquilla, Colombia.

3. Departamento de Pediatría, Facultad de Medicina, Universidad de Panamá. Panamá

4. Departamento de Pediatría, Facultad de Ciencias Médicas, Universidad Nacional de Asunción. Paraguay.

5. Centro de Recuperación Nutricional, Asociación Vínculo de Amor. San Salvador, El Salvador.

6. Departamento de Pediatría, Facultad de Medicina, Campus Centro, Universidad de Chile. Chile.

Correspondencia: Carlos Castillo-Durán, M.D., M.Sc. Departamento de Pediatría, Facultad de Medicina Campus Centro, Universidad

de Chile, Santa Rosa 1234, Santiago, Chile.E-mail: ccastd@med.uchile.cl

Recibido: 02/06/2014; Aceptado: 18/11/2014.

http://dx.doi.org/10.18004/ped.2015.abril.37-41

Los autores declaran que no existen conflictos de interés en el presente estudio. 
consumidos y estado nutricional de eutrófico, sobrepeso o desnutrición en los niños. Conclusiones: Los preescolares del grupo de estudio consumen una variedad limitada de los tipos de los alimentos localmente disponibles (sobre todo pescados y mariscos, hortalizas y frutas). También se observa un consumo rutinario de alimentos de alta densidad calórica y bebidas dulces.

Palabras clave: Consumo de alimentos, estado nutricional, preescolar, clase social.

\section{INTRODUCCIÓN}

El fenómeno reciente de la globalización social, política y cultural está ocasionando cambios fundamentales de los hábitos nutricionales en muchas comunidades. Los cambios resaltan la masiva disponibilidad de alimentos producidos en forma industrial, incluyendo la mayor accesibilidad a las comidas rápidas y las dulces, acompañadas de un flujo cada vez más alto de estos productos de los países desarrollados a los países en vías de desarrollo, observándose en contraparte un descenso en el consumo de alimentos naturales ${ }^{(1)}$. Esto constituye una globalización en los hábitos prevalentes de alimentación en ámbitos nacionales e internacionales ${ }^{(2)}$. En conjunto con estos cambios, se produce una pérdida de los conocimientos ancestrales de ciertos alimentos y preparaciones ${ }^{(3)}$.

En común con otras regiones, los países latinoamericanos comparten partes de una cultura de alimentación tradicional basada en la disponibilidad local de alimentos y los métodos de cocina y consumo ${ }^{(2)}$. En algunos países se debe considerar también la contribución de comunidades de ascendencia africana. Por sobre esta base, debemos agregar el impacto reciente de las empresas internacionales, por medio de los anuncios, en las cultura alimentaria y la industria de producción de alimentos de los países desarrollados ${ }^{(4)}$.

Disponer de un rango más variado de tipos de alimentos ha sido tradicionalmente una manera de satisfacer los requisitos para todos los nutrientes ${ }^{(5)}$. En contraste, consumir una escasa variedad de alimentos puede implicar un riesgo de deficiencias o excesos nutritivos $^{(3)}$.

Los años preescolares representan una etapa en que children in the study groups consume a limited variety of the individual foods locally available, particularly for fish/seafood, vegetables, and fruits. Routine consumption of calorie-dense foods and sweetened drinks was also found.

Keywords: Food consumption, nutritional status, preschool age, social class.

los niños empiezan a compartir la dieta familiar, así que la mayoría de los alimentos y métodos de preparación han sido incorporados a su dieta antes de la edad de cinco años. Existe poca información en cuanto a investigaciones previas sobre la magnitud de la variedad de los grupos de alimentos que consumen los preescolares latinoamericanos y a su vez dentro de cada grupo de alimentos.

Los objetivos del presente estudio fueron evaluar el número total y por grupos de alimentos, consumidos por los preescolares residentes en comunidades de bajos ingresos en ciudades seleccionadas de América Latina y evaluar la asociación entre el número total de variedad de alimentos (por grupos de alimentos) y el estado nutricional de los preescolares.

\section{METODOLOGÍA}

Estudio transversal, descriptivo, llevado a cabo entre el año 2010 y 2011. Fueron encuestadas 249 madres con ingresos económicos bajos con niños preescolares de entre tres y cinco años de edad de ambos sexos. Se alcanzó un total de 60 niños preescolares en cada ciudad. Los co-investigadores responsables en cada ciudad eligieron los niños de los centros de salud y jardines escolares en poblaciones de bajos ingresos en Santiago de Chile, San Salvador en El Salvador, la Ciudad de Panamá en Panamá, Asunción en el Paraguay y Barranquilla en Colombia.

En cada país, nutricionistas capacitadas indagaron a cada madre sobre el consumo de cada alimento por el niño. Usaron una entrevista semi-estructurada 
basada en una lista creada en cada ciudad para incluir todos los ingredientes disponibles en los mercados locales y agrupados en 11 grupos de alimentos: lácteos, cereales y tubérculos, hortalizas, frutas, mariscos, legumbres, carne y menudillos, carne procesada y huevos, azúcar y bebidas, grasas y aceites, condimentos. Se evaluó la variedad de consumo de cada grupo de alimentos consumidos por el niño. Se realizó antropometría en los preescolares (determinación del peso y de la talla de cada niño y el cálculo del índice de masa corporal, en condiciones estándar), según las normas de la Organización Mundial de la Salud (OMS) para su edad y sexo.

Los datos fueron reunidos en una plantilla de datos Excel. Se comprobó la bondad del ajuste para variables seleccionadas y ANOVA para comparar diferencias en la frecuencia del consumo de alimentos y grupos de alimentos en las ciudades estudiadas.

El estudio fue ejecutado en cumplimiento con las directrices de la Declaración de Helsinki y todos los procedimientos que involucraban participantes/pacientes humanos fueron aprobados por el Comité de Ética del Instituto de Nutrición y Tecnología de los Alimentos, Universidad de Chile. El consentimiento informado fue obtenido de todas las madres.

\section{RESULTADOS}

En la tabla 1 se muestran las características generales de los niños estudiados. La edad de los niños fue entre tres y cinco años y tuvieron como promedio cuatro meses de amamantamiento exclusivo. Hubo en la ciudad de Santiago y Asunción una mayor prevalencia de niños preescolares con sobrepeso. Sólo en la ciudad de Panamá y Barranquilla hubo preescolares con desnutrición.

En la tabla 2 se puede apreciar el promedio del número de alimentos consumidos por el hijo según las madres, ya sea en forma regular, por estación, o en ocasiones, relacionado a la cantidad de tipos de alimentos disponibles en cada localidad y por cada grupo de alimentos. El número de alimentos diferentes disponibles fue similar entre las ciudades de Santiago, San Salvador y Barranquilla (173, 177 y 171, respectivamente) y menor en la Ciudad de
Panamá y Asunción (129 y 111). Los grupos de alimentos con la mayor variedad disponible fueron en los pescados y mariscos, hortalizas y frutas. La menor proporción de alimentos consumidos en relación con los disponibles comercialmente se observó en cuanto a pescados y mariscos.

La tabla 3 ilustra el número total de variedad de los alimentos consumidos por el niño, según el estado nutricional y la ciudad.

Tabla 1. Características generales de niños pre escolares de ciudades latinoamericanas seleccionadas (Promedio \pm Desviación estándar (DE)).

\begin{tabular}{lcccccc}
\hline & Santiago & Barranquilla & \multicolumn{5}{c}{ San Salvador Panamá } & Asunción $\begin{array}{c}\text { Sig. } \\
\text { estad. }\end{array}$ \\
\hline $\mathrm{N}$ & 63 & 64 & 35 & 43 & 44 & \\
Edad (meses) & $50 \pm 6,2$ & $46 \pm 7,4$ & $52 \pm 8,9$ & $47 \pm 9,7$ & $51 \pm 8$ & $\mathrm{NS}$ \\
Sexo (M/F) & $27 / 36$ & $28 / 36$ & $21 / 14$ & $20 / 23$ & $19 / 25$ & $\mathrm{NS}$ \\
Amamantamiento (meses) & $4,1 \pm 2,7$ & $3,7 \pm 2,6$ & $3,0 \pm 2,2$ & $3,9 \pm 2,6$ & $3,3 \pm 2,5$ & $\mathrm{NS}$ \\
Estado nutricional & & & & & & \\
Eutróficos & 30 & 24 & 27 & 22 & 24 & \\
Sobrepeso & 33 & 21 & 7 & 9 & 20 & \\
Desnutridos & - & 19 & 1 & 12 & - & \\
\hline
\end{tabular}

Tabla 2. Consumo de alimentos (número promedio de alimentos consumidos) por grupos de alimentos en niños preescolares de bajos ingresos en ciudades latinoamericanas seleccionadas.

\begin{tabular}{lccccc}
\hline Grupo de Alimentos & Santiago & Barranquilla & San Salvador & Panamá & Asunción \\
\hline Lácteos & $4,8(9)$ & $4,6(10)$ & $6,1(10)$ & $5,2(8)$ & $4,3(6)$ \\
Cereales y tubérculos & $4,9(10)$ & $6,8(10)$ & $8,1(10)$ & $8,8(10)$ & $6,3(8)$ \\
\hline Hortalizas & $12,9(23)$ & $7,9(23)$ & $11,5(23)$ & $5,5(18)$ & $10,4(19)$ \\
Frutas & $17,1(33)$ & $16,1(36)$ & $21,2(36)$ & $10,5(20)$ & $11,9(20)$ \\
Grasas y aceites & $3,9(12)$ & $2,6(11)$ & $3,9(12)$ & $2,9(9)$ & $4,4(7)$ \\
Carne-vísceras & $4,3(17)$ & $5,5(16)$ & $3,9(16)$ & $4,2(9)$ & $4,8(9)$ \\
Pescados y mariscos & $2,3(28)$ & $4,2(28)$ & $5,0(22)$ & $3,3(20)$ & $2,3(7)$ \\
Huevo y carne procesada & $3,5(8)$ & $3,3(9)$ & $4,2(8)$ & $4,6(9)$ & $4,2(5)$ \\
Condimentos & $7,0(11)$ & $7,1(12)$ & $7,1(12)$ & $7,8(8)$ & $7,7(10)$ \\
Legumbres & $3,5(5)$ & $3,1(5)$ & $2,0(5)$ & $3,3(5)$ & $2,3(4)$ \\
\hline $\begin{array}{l}\text { Azúcar - bebidas } \\
\text { NOTA: Los resultados muestran el promedio en relación al número total de alimentos } \\
\text { diferentes disponibles en cada comunidad. }\end{array}$ & & & &
\end{tabular}

Tabla 3. Número de alimentos consumidos en cada grupo de alimentos por preescolares de bajos ingresos en ciudades latinoamericanas seleccionadas por estado nutricional de los niños $(\mathrm{x} \pm \mathrm{DE})$.

\begin{tabular}{lccccc}
\hline Estado Nutricional & $\begin{array}{c}\text { Santiago } \\
\mathbf{n = 6 3}\end{array}$ & $\begin{array}{c}\text { Barranquilla } \\
\mathbf{n = 6 4}\end{array}$ & $\begin{array}{c}\text { San Salvador } \\
\mathbf{n}=\mathbf{3 5}\end{array}$ & $\begin{array}{c}\text { Panamá } \\
\mathbf{n}=\mathbf{4 3}\end{array}$ & $\begin{array}{c}\text { Asunción } \\
\mathbf{n = 4 4}\end{array}$ \\
\hline Total & $75,4 \pm 10,3$ & $71,0 \pm 12,7$ & $86,4 \pm 14,9$ & $65,0 \pm 9,5$ & $71,0 \pm 13,9$ \\
Eutróficos & $\begin{array}{c}76,6 \pm 11,0 \\
(\mathrm{n}=30)\end{array}$ & $\begin{array}{c}75,0 \pm 14,8 \\
(\mathrm{n}=24)\end{array}$ & $\begin{array}{c}87,1 \pm 14,2 \\
(\mathrm{n}=27)\end{array}$ & $\begin{array}{c}65,5 \pm 9,1 \\
(\mathrm{n}=22)\end{array}$ & $\begin{array}{c}68,6 \pm 13,3 \\
(\mathrm{n}=24)\end{array}$ \\
Sobrepeso & $\begin{array}{c}76,6 \pm 11,0 \\
(\mathrm{n}=30)\end{array}$ & $\begin{array}{c}69,0 \pm 11,9 \\
(\mathrm{n}=21)\end{array}$ & $\begin{array}{c}86,2 \pm 16,7 \\
(\mathrm{n}=7)\end{array}$ & $\begin{array}{c}61,5 \pm 10,0 \\
(\mathrm{n}=9)\end{array}$ & $\begin{array}{c}73,9 \pm 14,3 \\
(\mathrm{n}=20)\end{array}$ \\
Desnutridos & - & $\begin{array}{c}67,6 \pm 9,9 \\
(\mathrm{n}=19)\end{array}$ & $\begin{array}{c}66 \\
(\mathrm{n}=1)\end{array}$ & $\begin{array}{c}66,4 \pm 10,3 \\
(\mathrm{n}=12)\end{array}$ & - \\
ANOVA $\mathrm{p}=$ & 0,3837 & 0,0969 & 0,8742 & 0,4808 & 0,2136 \\
\hline
\end{tabular}


El total del número de alimentos disponibles para uso rutinario en las comunidades estudiadas fue aproximadamente 170, con hallazgos similares en Santiago, Barranquilla y San Salvador. Hubo menor números de alimentos disponibles en Panamá y Asunción (Panamá: $n=123$; Asunción: $n=111$ ). El número de alimentos diferentes en el primer grupo de ciudades es similar al número disponible en los EE.UU. ${ }^{(6)}$. Se explica la situación en Asunción (Paraguay), con un consumo obviamente menor de mariscos por ser un país sin acceso al mar, mientras que en Panamá la deficiencia se observa principalmente en cuanto al consumo de una mayor variedad de frutas.

Se sabe que en las comunidades antiguas se consumían una amplia variedad de alimentos individuales, quizás entre 200 y $300^{(3)}$.

Se ha descrito una transición socio-cultural que conlleva un descenso dramático en el número de ingredientes alimentarios consumidos ${ }^{(3)}$. Muchos de los conocimientos relevantes acerca de ingredientes alimentarios comestibles se han perdido en las últimas generaciones (Ej. insectos, larvas y hongos comestibles), pero por otra parte el número total de alimentos ha tenido un incremento a causa de provisiones por la industria de alimentos y por el comercio internacional.

El número promedio de alimentos consumidos por los preescolares en relación a la disponibilidad en cada comunidad fue mayor en San Salvador (86) en relación a las otras comunidades: Panamá (65), Asunción y Barranquilla (71) y Santiago (75).

La transición de un consumo de alimentos basado en la caza y recolección y agricultura básica a un estilo de consumo de las comunidades desarrollados está asociada a la pérdida del consumo tradicional de ciertos alimentos y su sustitución por otros más apetitosos y más fáciles de consumir, y que viene firmemente vinculada a una reducción en deficiencias y enfermedades nutricionales y a la vez con un mayor incidencia de enfermedades asociadas al exceso del consumo de algunos nutrientes ${ }^{(7-13)}$.

En cuanto al consumo de mariscos, el costo no parece ser un factor importante, debido a los precios relativamente asequibles en las ciudades con acceso directo al mar. Factores culturales, tales como percepciones relacionadas a su inocuidad para el consumo (por ser perecederos o por el contenido de metil-mercurio), podrían figurar en las decisiones familiares.

En cuanto a las hortalizas, hubo una amplia disponibilidad en todas las ciudades. Sin embargo, frecuentemente los niños y las familias latinoamericanas de bajos ingresos, no cuentan con una rutina estándar para su cocción y existe el riesgo de infecciones por consumo de hortalizas crudas preparadas sin los cuidados higiénicos adecuados ${ }^{(14-17)}$.

Disponer de la variedad máxima de alimentos disponibles en la comunidad es un aspecto importante para evitar deficiencias nutritivas en las comunidades con riesgo nutricional, y también tiene un papel en evitar enfermedades relacionadas a la malnutrición, en particular la obesidad. Empezando en los primeros años de la vida, consumir una variedad de alimentos tiene un papel importante en los órganos sensoriales y de aprendizaje asociados con el apetito y el consumo de alimentos ${ }^{(18)}$. En resumen, los niños preescolares de niveles socioeconómicos bajos en las ciudades seleccionadas consumen un rango restringido de los alimentos disponibles en sus comunidades, un hallazgo que fue similar en todos los países estudiados. En general, se detecta en niños pre escolares un consumo de menor variedad en el grupo de hortalizas, frutas, pescados y mariscos. No hubo una diferencia significativa en el número de alimentos consumidos en cada grupo de alimentos asociado al estado de nutrición de los niños.

\section{RECONOCIMIENTOS}

El estudio presentado fue financiado por recursos propios. Agradecemos sinceramente la colaboración de Victoria Valdés (dietista registrada, Panamá), Dra. Paola Renna y Sara Móngelos (Pediatra y Licenciada en Nutrición, registradas, Paraguay) para la implementación de las encuestas locales. 


\section{REFERENCIAS}

1. Popkin BM. Nutritional patterns and transition. Popul Dev Rev. 1993;19:138-57.

2. Romaguera D, Samman N, Rossi A, Miranda C, Pons A, Tur JA. Dietary patterns of the Andean population of Puna and Quebrada of Humahuaca, Jujuy, Argentina. Br J Nutr. 2008;99:390-97.

3. Robson JRK, Wadsworth GR. The health and nutritional status of primitive populations. Ecol Food Nutr. 1977;6:187-202.

4. Batis C, Hernández-Barrera L, Barquera S, Rivera JA, Popkin BM. Food acculturation drives dietary differences among Mexicans, Mexican Americans, and Non-Hispanic whites. J Nutr. 2011;141:1898-1906.

5. Mark L, Wahlqvist ML. Diversification in indigenous and ethnic food culture. Forum Nutr Karger. 2005;57:52-61.

6. United States Department of Agriculture. Daily food plan for preschoolers [Internet]. Alexandria: United States Department of Agriculture. USDA Center for Nutrition Policy and Promotion [actualizado 2012 mar 05; citado 2012 jun 20]. Disponible en: http://www.choosemyplate. gov/food-groups/grains.html

7. Avena NM, Gold MS. Variety and hyperpalatability: are they promoting addictive overeating?. Am J Clin Nutr. 2011;94:367-68.

8. Monteiro CA, Conde WL, Popkin BM. Is obesity replacing or adding to undernutrition? evidence from different social classes in Brazil. Public Health Nutr. 2002;5:105-112.

9. Wang Y, Monteiro C, Popkin BM. Trends of obesity and underweight in older children and adolescents in the United States, Brazil, China, and Russia. Am J Clin Nutr. 2002;75:971-77.
10. Organización Mundial de la Salud. Dieta, nutrición y prevención de enfermedades crónicas: informe de una consulta mixta de expertos OMS/FAO. Ginebra: Organización Mundial de la Salud; 2003.

11. Albala C, Vio F. Epidemiological transition in Latin America: the case of Chile. Publ Health. 1995;109:431-42.

12. Martorell R, Kettel Khan L, Hughes ML, GrummerStrawn LM. Overweight and obesity in preschool children from developing countries. Int J Obes Relat Metab Disord. 2000;24:959-67.

13. Del Rea S, Fajardo Z, Solano L, Páez M, Sánchez AM. Patrón de consumo de alimentos en niños de una comunidad urbana al norte de Valencia, Venezuela. Arch Latinoam Nutr. 2005; 55:279-86.

14. Nicklas TA, Baranowski T, Baranowski J. Family and child-care provider influences on preschool children's fruit, juice, and vegetable consumption. Nutr Rev. 2001;59:224-35

15. Heather P, Nicklas T. A review of family and social determinants of children's eating patterns and diet quality. J Am College Nutr. 2005;24:83-92.

16. Mahaffey KR, Sunderland EM, Chan HM. Balancing the benefits of $n-3$ polyunsaturated fatty acids and the risks of methyl mercury exposure from fish consumption. Nutr Rev. 2011;69:493-508.

17. Lanata CF. Studies of food hygiene and diarrhoeal disease. Int J Environ Health Res. 2003;13(Suppl 1):S175-183.

18. Rigal N. Food diversification and taste building. Arch Pediatr. 2010;17(Suppl5):S208-212. 